\title{
Outcomes of Cardiopulmonary Resuscitation and its Predictors in Hospitalized Patients

\author{
Meisam Moezzi ${ }^{1}$, Golshan Afshari2 ${ }^{*}$, Fakher Rahim ${ }^{3}$, Meysam Alavian ${ }^{2}$, Maryam Bani- \\ torfi', Saeed Hesam ${ }^{4}$ and Nasrin Fatemi ${ }^{2}$
}

${ }^{1}$ Department of Emergency Medicine, Ahvaz Jundishapur University of Medical Sciences, Ahvaz, Iran
${ }^{2}$ Golestan Hospital Clinical Research Development Unit, Ahvaz Jundishapur University of Medical Sciences, Iran

${ }^{3}$ Research Center of Thalassemia and Hemoglobinopathy, Health Research Institute, Ahvaz Jundishapur University of Medical Sciences, Iran

${ }^{4}$ Department of Biostatistics, Ahvaz Jundishapur University of Medical Sciences, Ahvaz, Iran

*Corresponding author: Golshan Afshari, Golestan Hospital Clinical Research Development Unit, Ahvaz Jundishapur University of Medical Sciences, Ahvaz, Iran, Tel: +989166004225

\begin{abstract}
Objective: Cardiopulmonary resuscitation (CPR) has been a frequently performed medical intervention that increases the chance of survival of a person strickenby cardiac arrest, and there is a great value of diversity in the rate of successful rehabilitation in societies.

Design: A retrospective observational study was carried out. The medical records of all in-hospital and out-hospital cardiac arrest patients who underwent CPR.

Patients or participants: A total of 587 people with which underwent CPR during the 2-year period 2017 to 2018; using a designed form.

Main variables of interest: Includes demographic information, the ward which CPR was committed, hospitalization, the delay before the onset of CPR, time of the day, and so on, were obtained.

Results: There was no significant difference in the success rate of CPR between men and women. The comparison of age groups revealed a significant difference between the success rates of CPR in the age of 14-64 with the group above 64 . The analysis of the data revealed no significant difference between CPR success rates in various seasons. Investigating the occurrence of cardiac arrest and its success rate at the hospital shifting show a significant difference between the success rate of CPR in the morning shift with the evening shift and night. The dual comparison revealed a significant difference in the success rate of CPR only between the emergency department and intensive care units.
\end{abstract}

Conclusions: The current study revealed a significant difference in age group, and location and so did not show any significant success rate of CPR in the presence of witnesses, location of cardiac arrest, season and gender.

\section{Keywords \\ Cardiopulmonary resuscitation, Cardiac arrest}

\section{Introduction}

Cardiopulmonary resuscitation (CPR) has been a frequently performed medical intervention that increases the chance of survival of a person suffering from cardiac arrest or heart failure. A group of researches revealed a survival rate ranging from $6 \%$ to $43 \%$ in cancer and monitored bed patients $[1,2]$. CPR involves actions taken to restore the function of the two vital organs of the body, heart, and brain in a person who has lost consciousness. Several factors were claimed to be responsible for such variation, including various selection criteria, different settings, and problems with definitions of common variables [3]. Cardiac arrest may be due to an underlying medical condition such as heart disease, pulmonary dysfunction, poisoning, cancers, a variety of strokes, electrocution, drowning, frostbite, etc. [4].

According to the American Heart Association, nearly $95 \%$ of the sudden cardiac deaths die before they reach

Citation: Moezzi M, Afshari G, Rahim F, Alavian M, Banitorfi M, et al. (2020) Outcomes of Cardiopulmonary Resuscitation and its Predictors in Hospitalized Patients. Clin Med Rev Case Rep 7:309. doi. org/10.23937/2378-3656/1410309

Accepted: May 11, 2020: Published: May 13, 2020

Copyright: (c) 2020 Moezzi M, et al. This is an open-access article distributed under the terms of the Creative Commons Attribution License, which permits unrestricted use, distribution, and reproduction in any medium, provided the original author and source are credited. 
the hospital, and if after cardiac arrest, CPR did not provide, for every one minute delay in starting the CPR, 7-10 percent increases in the risk of death [5]. Today, cardiopulmonary resuscitation can be done in two basic forms basic life support (BLS) and advanced life support (ALS) [6]. With the above explanations, one of the greatest challenges in performing CPR is time to start, so that even a minute delay at the start of resuscitation reduces the likelihood of success. The importance of time is such that, when CPR begins with a delay of 10 minutes, despite the success of heart resuscitation, brain death will occur [7]. Any major issue in prognosis and outcomes in cardiac arrest is the location of the patient at the time of $C P R$, time of starting CPR, underlying disease, initial electrocardiogram (EKG) rhythm, proper defibrillation and most importantly quality of CPR [8].

Though performing CPR has made strong progress in recent years, limited data on the outcomes of CPR from low and middle-income countries exist [8-11]. Thus, various settings and resources in low and middle-income countries have the potential to affect the eventual outcome of CPR [12]. This study aims to determine the possible success rate of CPR in patients who had a cardiac arrest at a referral hospital in southwestern Iran, as well as to provide a conceptual framework based on CPR outcomes.

\section{Patients and Method}

\section{Study design and population}

This retrospective study analyzed the medical records of all in-hospital and out-hospital cardiac arrest patients who underwent CPR during the 2-year period 2017 to 2018, using forms designed by the Ministry of Health for recording CPR data (checklist 1). This study was approved by the Ethical Committee of -- University of Medical Sciences.

\section{Setting}

This hospital was a 660-bed facility with an Emergency Department comprising of 50 monitored beds and two resuscitation beds. During the study period, more than 80,000 patients underwent either emergency department visits or admitted to the hospital, of which 578 patients either present with cardiac arrest or go into arrest during the course of a hospital stay.

\section{Inclusion criteria}

Patients (age $>10$ years) who had sustained either an in- or out-of-hospital cardiac arrest and were brought to the ED for treatment, or who underwent CPR between January 2017 and June 2018 anywhere in the hospital.

\section{Exclusion criteria}

Patients who were reported dead on hospital arrival and those who had an advance directive of no CPR (NCPR), or those with missing records or incomplete data were excluded.

\section{Data collection}

The information includes demographic information, the ward which CPR was committed, hospitalization, the delay before the onset of CPR, time of the day, and so on were obtained. According to the American Heart Association, successful resuscitation or ROSC is defined for all rhythms as the restoration of an impressive spontaneous rhythm that results in more than an occasional gasp, fleeting palpated pulse, or arterial waveform.

\section{Statistical analysis}

Data was presented as the number and percentage of patients with return of spontaneous circulation (ROSC) after CPR, subjects alive for more than $24 \mathrm{~h}$, and those who had survived to discharge (STD). To compare the difference of the seasons, compared the number of patients and the success rates of CPR in different seasons of the year. To describe the quantitative variables, the mean and standard deviations were used to describe qualitative variables from frequency and percentage. For data analysis, simple logistic regression was used. The data were analyzed using SPSS version 21.0 (IBM, Chicago, IL), and $p$-value $<0.05$ was considered significant.

\section{Results}

The first set of analyses examined the number of people who were receiving CPR based on gender. Overall, 587 CPR operations were carried out on 361 men (61\%), 231 women (39\%), of which 152 (25.8\%) successful, 435 other operations (73.8\%) were unsuccessful. There was no significant difference $(p=0.271)$ in the success rate of CPR between men and women (Table 1 and Figure 1). The mean age of patients who underwent CPR was $59 \pm 22$ years, of which 31 cases (5.5\%) were 10 to 14 , and 264 (46.6\%) were over the age of 14 to 64 , and 271 (47.9\% were above 64 -years-old. There was a significant difference between CPR in three age groups 10 to 14 -years-old, 14 to 64 , and older than 64

Table 1: Population and event characteristics.

\begin{tabular}{|l|l|l|l|l|l|}
\hline \multicolumn{2}{|l|}{ Characteristic } & \multicolumn{2}{l|}{ CPR (\% Within CPR) } & \multicolumn{2}{l|}{} \\
\cline { 3 - 6 } & Successful, n (\%) & Unsuccessful, n (\%) & Total, n (\%) & P-Value \\
\hline \multirow{2}{*}{ Gender } & Male & $87(57.2 \%)$ & $271(62.3 \%)$ & $358(61 \%)$ & 0.271 \\
\cline { 2 - 6 } & Female & $65(42.8 \%)$ & $164(37.7 \%)$ & $229(39 \%)$ & \\
\cline { 2 - 6 } & Total & $\mathbf{1 5 2}$ & $\mathbf{4 3 5}$ & $\mathbf{5 8 7}$ & \\
\hline
\end{tabular}




\begin{tabular}{|c|c|c|c|c|c|}
\hline \multirow[t]{4}{*}{ Age } & $10-14$ & $7(4.8 \%)$ & $24(5.7 \%)$ & $31(5.5 \%)$ & \multirow[t]{4}{*}{0.002} \\
\hline & $14-64$ & $85(57.8 \%)$ & $179(42.7 \%)$ & $264(46.6 \%)$ & \\
\hline & $\geq 64$ & $55(37.4 \%)$ & $216(51.6 \%)$ & $271(47.9 \%)$ & \\
\hline & Total & 31 & 271 & 566 & \\
\hline \multirow[t]{5}{*}{ Season } & Spring & $46(30.3 \%)$ & $140(32.2 \%)$ & $186(31.7 \%)$ & \multirow[t]{5}{*}{0.495} \\
\hline & Summer & $25(16.4 \%)$ & $51(11.7 \%)$ & $76(12.9 \%)$ & \\
\hline & Autumn & $21(13.8 \%)$ & $69(15.9 \%)$ & $90(15.3 \%)$ & \\
\hline & Winter & $60(39.5 \%)$ & $175(40.2 \%)$ & $235(40 \%)$ & \\
\hline & Total & 152 & 435 & 587 & \\
\hline \multirow[t]{3}{*}{ Location } & Inside the hospital & $137(92.6 \%)$ & $409(94.2 \%)$ & $546(93.8 \%)$ & \multirow[t]{3}{*}{0.466} \\
\hline & Out of hospital & $11(7.4 \%)$ & $25(5.8 \%)$ & $36(6.2 \%)$ & \\
\hline & Total & 148 & 434 & 582 & \\
\hline \multirow[t]{4}{*}{ Shift } & Morning & $51(33.6 \%)$ & $100(23 \%)$ & $151(25.7 \%)$ & \multirow[t]{4}{*}{0.037} \\
\hline & Afternoon & $32(21.1 \%)$ & $108(24.8 \%)$ & $140(23.9 \%)$ & \\
\hline & Night & $69(45.4 \%)$ & $227(52.2 \%)$ & $296(50.4 \%)$ & \\
\hline & Total & 152 & 435 & 587 & \\
\hline \multirow[t]{4}{*}{ Ward } & Emergency ward & $57(38.5 \%)$ & $212(49 \%)$ & $269(46.3 \%)$ & \multirow[t]{4}{*}{0.041} \\
\hline & General ward & $71(48 \%)$ & $185(42.7 \%)$ & $256(44.1 \%)$ & \\
\hline & Intensive care unit & $36(8.3 \%)$ & $20(13.5 \%)$ & $56(9.6 \%)$ & \\
\hline & Total & 148 & 433 & 581 & \\
\hline \multirow[t]{3}{*}{ Witness } & Present & $36(87.8 \%)$ & $115(85.8 \%)$ & $151(86.3 \%)$ & \multirow[t]{3}{*}{0.9} \\
\hline & Not present & $5(12.2 \%)$ & $19(14.2 \%)$ & $24(13.7 \%)$ & \\
\hline & Total & 41 & 134 & 175 & \\
\hline
\end{tabular}

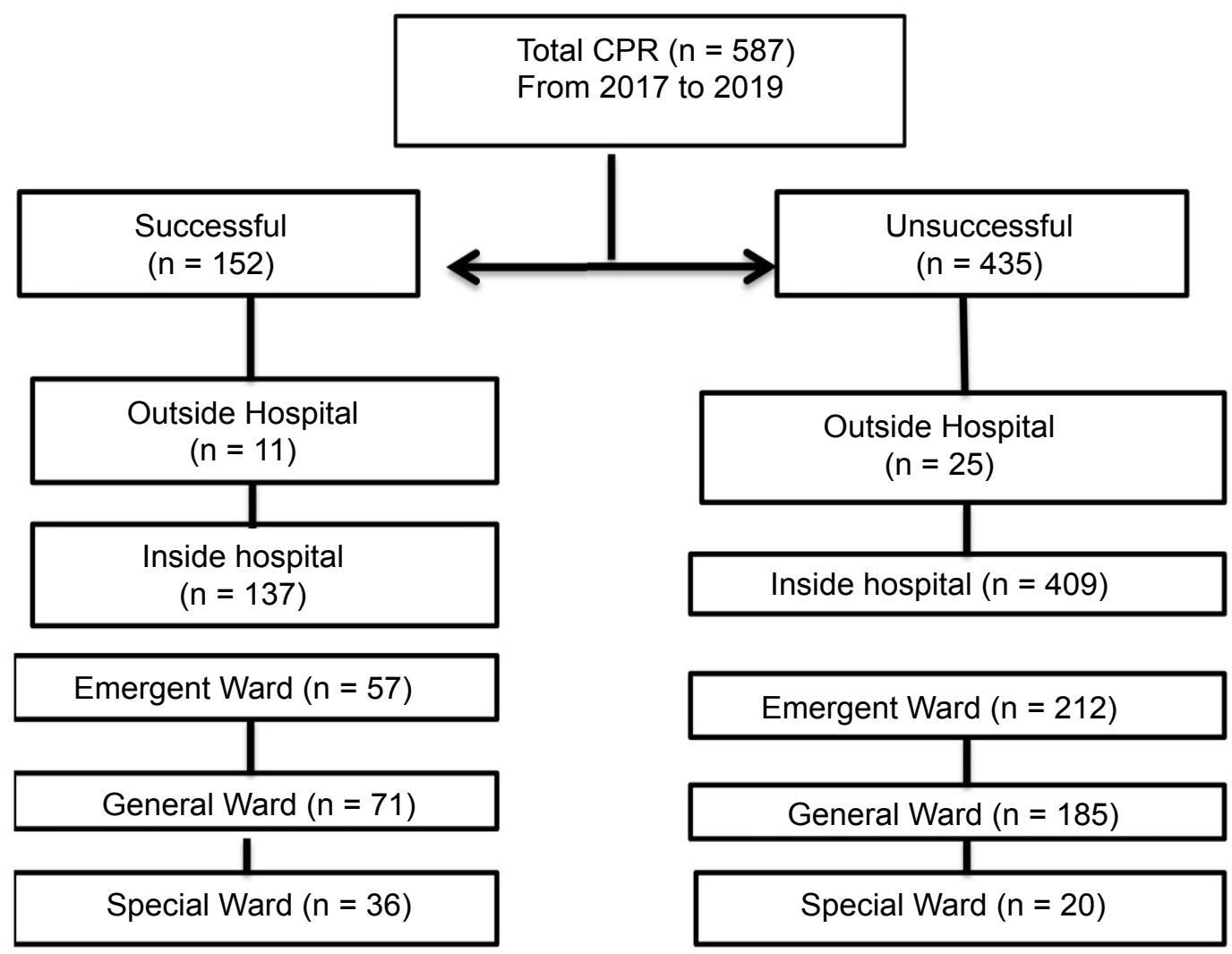

Figure 1: Utstein-style flow chart of patient and outcomes. 
( $p=0.006)$. The comparison of age groups revealed a significant difference only between the success rate of CPR in the age group of 14-64 with the group above 64 $(p=0.002)$.

The results indicated that 186 (31.7\%) CPR were performed in the spring with a $30.3 \%$ success rate, in the summer in $76(12.8 \%)$ cases with a $16.4 \%$ success rate, in the Autumn in $90(15.2 \%)$ case with $13.8 \%$ success rate and finally in the winter in $235(40 \%)$ cases with a $39.5 \%$ success rate. According to the results, most of the patient was in the winter, and the highest percentage of success was in the same season; however, data analysis revealed no significant difference between CPR success rate in various seasons. Since the climatic conditions of the study area are tropical and there are practically no distinct four seasons, another analysis was performed and comparing the first six months of the year (hot months) with the second six months of the year (cooler months). The second analyses show no significant differences.

Following this, the results of the site of CPR showed that $94.2 \%$ of cardiac arrests happened in the hospital, albeit there were no significant differences between CPR success rates between outside and inside the hospital (Table 1). Investigating the occurrence of cardiac arrest and its success rate at the hospital's shifting showed that there was a significant difference between CPR success rate in three different shifts $(p=0.037)$. Using the LSD follow-up test, we compared shifts, which showed a significant difference between the success rate of CPR in the morning shift with the evening shift ( $p$ $=0.034)$ and night $(p=0.017)$. Also, there was no significant difference between the success rates of CPR operation in the evening shift with night shifts.

Investigating the CPR in different sites of the hospital revealed that of the 581 operations carried out, 269 operations (46.3\%) were carried out in the emergency department, 256 other operations (44\%) in the general wards and 56 cases (9\%) in intensive care units, and in the six cases, the site that the CPR was taking place was not reported. The results of the analysis showed a significant difference between the rate of CPR's success on different sites $(P=0.041)$. The dual comparison revealed a significant difference in the success rate of $C P R$ only between the emergency department and intensive care units $(p=0.023)$.

The results of the presence of a witness (therapist) at the moment of the cardiac arrest showed that of 175 cases, the witness in the 152 cases was at the patient's bedside, and about 24 cases, there were no witnesses. Data analysis revealed that was no significant difference between the success rates of CPR operation in the presence of the witness and the absence of the witness ( $P$ $=0.09$ ).

It is necessary to mention that several recording forms of recovery have not been filled completely, and we have lost some data. In the age variable, 20 forms, in the witness 412 forms, in ward six and in the location 5 forms were incomplete.

In summary, these results show that in the current study, the difference in age, wards, and shift was significant.

\section{Discussion}

The present study was designed to determine the effect of different effective factors on the CPR success rate, which showed that resuscitation was not significantly different between men and women. The results of this study are compatible with the findings of previous work in Iran, China, and America, which reported that the CPR success rate is not affected by the gender of the patients $[10,13-15]$. Another important finding was that the success rate of CPR in the age group of 1464 was higher than that of the group above 64 years. This finding confirms the association between aging and the success rate of CPR, as has already been reported in previous studies $[16,17]$. In contrast, another study on the association between the age and survival after cardiac arrest at the outside of the hospital and reported that although age is related to the survival of people after cardiac arrest, however, age alone does not predict the outcome of CPR and is, in fact, a poor indicator of survival [18].

Interestingly, a group of researchers examined the relationship between high and low air temperatures and outside hospital cardiac arrest with a heart attack cause. The results of the study indicated that the occurrence of cardiac arrest outside the hospital is associated with low air temperature. In addition, the occurrence of cardiac arrest in people aged 80 years or older was associated with high air temperatures [19]. Contrary to expectations, this study did not find a significant difference between CPR success rates at distinct seasons.

The prevalence of outside the hospital cardiac arrest is more than 300,000 people in the United States per year and is the third main cause of death, and an evaluated 290,000 in-hospital cardiac arrests happen each year in the United States [20,21]. Moreover, during the 2010 evaluation, the in-hospital mortality rate after cardiac arrest was reported at the level of $81.6 \%$ in Great Britain [22], 77.7\% in the United States [23], 74\% in Poland, 59\% in Italy [24] and $70 \%$ in Sweden. In the present study, in-hospital and out-hospital mortality rates after cardiac arrest were $74.9 \%$ and $69.4 \%$, respectively. Further, it can be said that we have similar results with the articles mentioned above.

Although the shortage of manpower and fatigue of therapists in the night shift affects the delivery of optimal services have been addressed previously, no data was found on the association between working shift and the success of CPR and the quality of services $[25,26]$. Contrary to expectations, the current study finds a sig- 


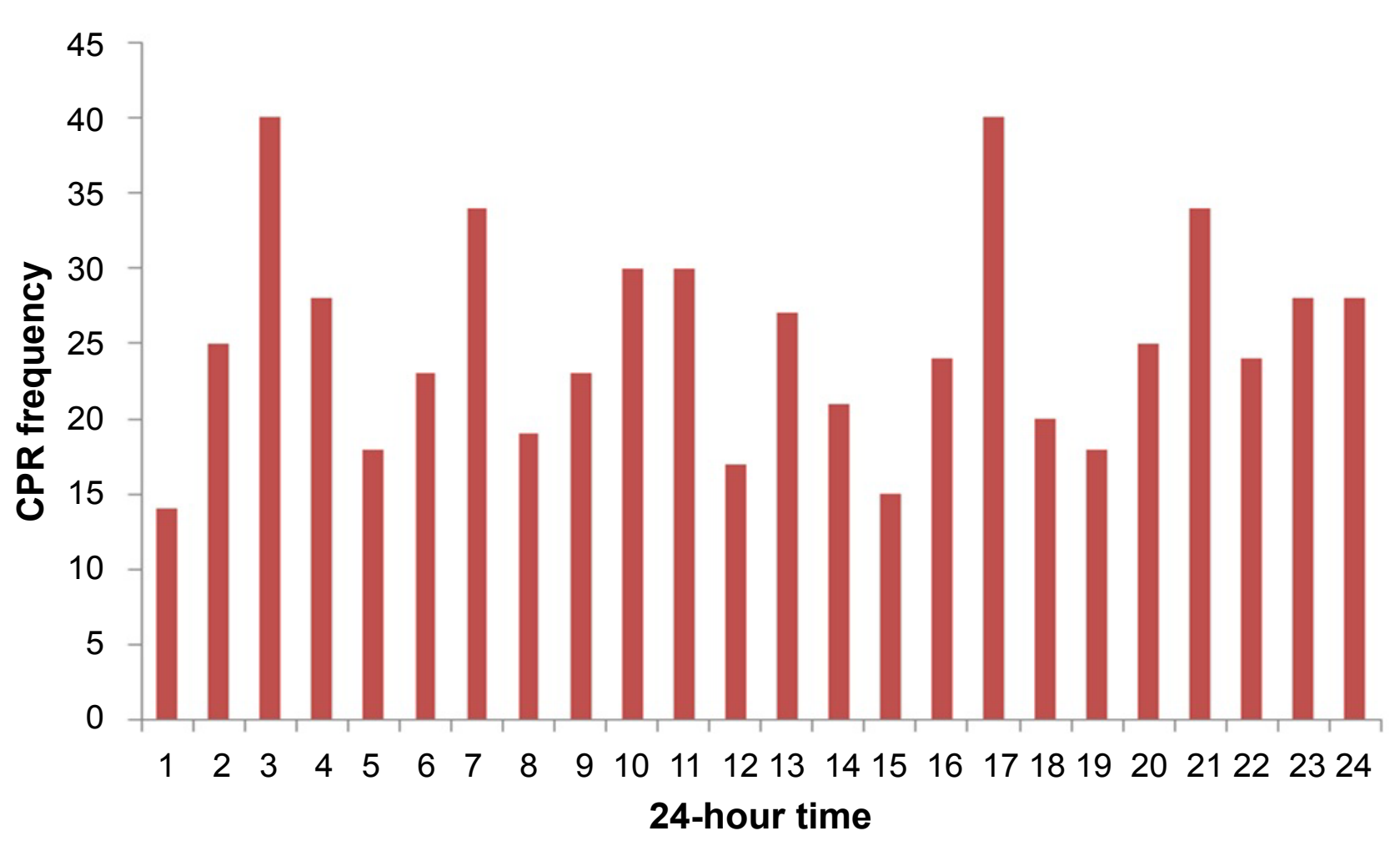

Figure 2: Prevalence of arrest by the hour of occurrence.

nificant difference between CPR success rate and work shift' although, some studies have reported a relationship between long working hours and reverse outcomes in health care $[27,28]$.

As you can see in Figure 2, the peak of cardiac arrest occurred at 3 am and $4 \mathrm{pm}$. Recently, several studies on the circadian clock revealed that there is a linked between The pathogenesis of heart disease in response to adverse stress, such as ischemia/reperfusion and cardiomyocytes circadian clock $[29,30]$.

Further statistical tests revealed that in the current experiment, there is a significant difference in the success rate of CPR only between the emergency department and intensive care units. Although it was not possible to investigate the significant relationships of different wards on the success rate of CPR, A number of issues were identified that the percentage of survival after CPR in ICUs is higher than in emergency situations [31]. But various studies have acknowledged that advanced life support is provided by the cardiac arrest team, and the timely and correct use of a defibrillator are the most important factors in the survival of patients [32,33].

However, there have been no sufficient controlled studies that compare differences in the effects of the presence or absence of witnesses at the moment of cardiac arrest. The results of this study did not show any significant increase in the success rate of CPR in the presence of witnesses. This may be explained by the fact that there is not a complete record of the presence or absence of witnesses at the moment of cardiac arrest in the majority of CPR forms and need to be further explored in the future. Although information about the effects of the presence of a witness in the moment of cardiac arrest is limited, a study in 2013 reported that in-hospital cardiac arrest had a higher chance of survival after discharge in the presence of the witness, comparable ones with monitoring and revealed that monitored cases had a lower rate of survival to hospital discharge [34].

\section{Limitation}

The time interval for CPR announcement to start operations in all registries reported as zero. Of course, this claim is a matter of debate, and we report it as a limitation of the current study.

\section{Conclusion}

This is the first epidemiological study of cardiac arrest at this center. The results of this study did not show any significant increase in the success rate of CPR in the presence of witnesses, location of cardiac arrest, season, and gender. Despite the differences in cases and diagnosis of the disease and the reason for hospitalization, the main factors contributing to the success rate of CPR were age, work shift, and hospital wards. However, we need to go into more detail about the probable background diseases, past medical history, and, most importantly, the quality of CPR factors that influence CPR success in future reviews.

\section{Contribution of the Authors}

\section{Supplementary}

The checklist is uploaded as a supplementary file. 


\section{Acknowledgment}

This paper was derived from a research project approved by the Ahvaz Jundishapur University of Medical Sciences. Our thanks and appreciations go to the member of the Clinical Research Development Unit and Quality Improvement Unit of Ahvaz Jundishapur University of Medical Sciences.

\section{Disclosure and conflicts of interest}

The authors declare no conflict of interest.

\section{References}

1. Reisfield GM, Wallace SK, Munsell MF, Webb FJ, Alvarez $E R$, et al. (2006) Survival in cancer patients undergoing in-hospital cardiopulmonary resuscitation: a meta-analysis. Resuscitation 71: 152-160.

2. Herlitz J, Bang A, Aune S, Ekstrom L, Lundstrom G, et al. (2001) Characteristics, and outcome among patients suffering in-hospital cardiac arrest in monitored and non-monitored areas. Resuscitation 48: 125-135.

3. Perkins GD, Jacobs IG, Nadkarni VM, Berg RA, Bhanji F, et al. (2015) Cardiac arrest and cardiopulmonary resuscitation outcome reports: update of the Utstein Resuscitation Registry Templates for Out-of-Hospital Cardiac Arrest: A statement for healthcare professionals from a task force of the International Liaison Committee on Resuscitation (American Heart Association, European Resuscitation Council, Australian and New Zealand Council on Resuscitation, Heart and Stroke Foundation of Canada, InterAmerican Heart Foundation, Resuscitation Council of Southern Africa, Resuscitation Council of Asia); and the American Heart Association Emergency Cardiovascular Care Committee and the Council on Cardiopulmonary, Critical Care, Perioperative and Resuscitation. Circulation 132: 1286-1300.

4. Kuch M, Janiszewski M, Mamcarz A, Cudnoch-Jedrzejewska A, Dluzniewski M (2009) Major adverse cardiac event predictors in survivors of myocardial infarction with asymptomatic left ventricular dysfunction or chronic heart failure. Med Sci Monit 15: Ph40-Ph48.

5. American Heart Association hwzc-mupsctrchc-f-plao.

6. Kurz MC, Schmicker RH, Leroux B, Nichol G, Aufderheide TP, et al. (2018) Advanced vs. Basic life support in the treatment of out-of-hospital cardiopulmonary arrest in the resuscitation outcomes consortium. Resuscitation 128: 132-137.

7. Song F, Sun S, Ristagno G, Yu T, Shan Y, et al. (2011) Delayed high-quality CPR does not improve outcomes. Resuscitation 82: S52-S55.

8. Pembeci K, Yildirim A, Turan E, Buget M, Camci E, et al (2006) Assessment of the success of cardiopulmonary resuscitation attempts performed in a Turkish university hospital. Resuscitation 68: 221-229.

9. Hajbaghery MA, Mousavi G, Akbari H (2005) Factors influencing survival after in-hospital cardiopulmonary resuscitation. Resuscitation 66: 317-321.

10. Hajzargarbashi E, Omidi E, Esmailian M (2019) Correlation of Patients' Baseline Characteristics with Success Rate of Cardiopulmonary Resuscitation; a Cross-Sectional Study. Advanced Journal of Emergency Medicine 3: e6.

11. Suraseranivongse $S$, Chawaruechai $T$, Saengsung $P$, Komoltri C (2006) Outcome of cardiopulmonary resuscitation in a 2300 -bed hospital in a developing country. Resuscita- tion 71: 188-193.

12. Moosajee US, Saleem SG, Iftikhar S, Samad L (2018) Outcomes following cardiopulmonary resuscitation in an emergency department of a low- and middle-income country. International Journal of Emergency Medicine 11: 40.

13. Stotts MJ, Hung KW, Benson A, Biggins SW (2014) Rate and predictors of successful cardiopulmonary resuscitation in end-stage liver disease. Dig Dis Sci 59: 1983-1986.

14. Zhang C, Wang Y, Liu H, Hao A, Xun J, et al. (2017) Comparative study for effects of bare-handed CPR with Thumper cardiopulmonary resuscitator CPR in emergency department based on "the Utstein style". Zhonghua Wei Zhong Bing Ji Jiu Yi Xue 29: 937-939.

15. Dicker B, Conaglen K, Howie G (2018) Gender and survival from out-of-hospital cardiac arrest: a New Zealand registry study. Emergency Medicine Journal 35: 367-371.

16. Winther-Jensen M, Kjaergaard J, Hassager C, Bro-Jeppesen J, Nielsen N, et al. (2015) Resuscitation and post resuscitation care of the very old after out-of-hospital cardiac arrest is worthwhile. International Journal of Cardiology 201: 616-623.

17. Snyder JE, Loschner AL, Kepley HO (2010) The effect of patient age on perceived resuscitation outcomes by practitioners. North Carolina Medical Journal 71: 199-205.

18. Andersen LW, Bivens MJ, Giberson T, Giberson B, Mottley $\mathrm{JL}$, et al. (2015) The relationship between age and outcome in out-of-hospital cardiac arrest patients. Resuscitation 94: 49-54.

19. Yamazaki S, Michikawa T (2017) Association between high and low ambient temperature and out-of-hospital cardiac arrest with cardiac etiology in Japan: A case-crossover study. Environ Health Prev Med 22: 60.

20. Milan M, Perman SM (2016) Out of hospital Cardiac Arrest: $A$ current review of the literature that informed the 2015 American Heart Association Guidelines update. Curr Emerg Hosp Med Rep 4: 164-171.

21. Andersen LW, Holmberg MJ, Berg KM, Donnino MW, Granfeldt A (2019) In-Hospital Cardiac Arrest: A Review. JAMA 321: 1200-1210.

22. Nolan JP, Soar J, Smith GB, Gwinnutt C, Parrott F, et al. (2014) Incidence and outcome of in-hospital cardiac arrest in the United Kingdom National Cardiac Arrest Audit. Resuscitation 85: 987-992.

23. Roger VL, Go AS, Lloyd-Jones DM, Adams RJ, Berry JD, et al. (2011) Heart disease and stroke statistics--2011 update: a report from the American Heart Association. Circulation 123: e18-e209.

24. Radeschi G, Mina A, Berta G, Fassiola A, Roasio A, et al. (2017) Incidence and outcome of in-hospital cardiac arrest in Italy: a multicentre observational study in the Piedmont Region. Resuscitation 119: 48-55.

25. Zafari AM, Zarter SK, Heggen V, Wilson P, Taylor RA, et al. (2004) A program encouraging early defibrillation results in improved in-hospital resuscitation efficacy. Journal of the American College of Cardiology 44: 846-852.

26. Citolino CM, Santos ES, Silva Rde C, Nogueira Lde S (2015) Factors affecting the quality of cardiopulmonary resuscitation in inpatient units: perception of nurses. Revista da Escola de Enfermagem da USP 49: 908-914.

27. Dembe AE, Delbos R, Erickson JB (2009) Estimates of injury risks for healthcare personnel working night shifts and long hours. Quality \& Safety in Health Care 18: 336-340. 
28. Bae SH, Fabry D (2014) Assessing the relationships between nurse work hours/overtime and nurse and patient outcomes: systematic literature review. Nursing Outlook 62: 138-156.

29. Durgan DJ, Young ME (2010) The cardiomyocyte circadian clock: emerging roles in health and disease. Circ Res 106: 647-658.

30. Jeyaraj D, Haldar SM, Wan X, McCauley MD, Ripperger JA, et al. (2012) Circadian rhythms govern cardiac repolarization and arrhythmogenesis. Nature 483: 96-99.

31. Vafaei A, Shams Akhtari A, Heidari K, Hosseini S (2018) Quality of cardiopulmonary resuscitation in emergency department based on the AHA 2015 guidelines: A Brief Report. Emerg (Tehran) 6: e46.
32. Sandroni C, Ferro G, Santangelo S, Tortora F, Mistura L, et al. (2004) In-hospital cardiac arrest: survival depends mainly on the effectiveness of the emergency response. Resuscitation 62: 291-297.

33. Assar S, Husseinzadeh M, Nikravesh AH, Davoodzadeh $\mathrm{H}$ (2016) The Success Rate of Pediatric In-Hospital Cardiopulmonary Resuscitation in Ahvaz Training Hospitals. Scientifica 2016: 8.

34. Chon GR, Lee J, Shin Y, Huh JW, Lim CM, et al. (2013) Clinical outcomes of witnessed and monitored cases of in-hospital cardiac arrest in the general ward of a university hospital in Korea. Respiratory Care 58: 1937-1944. 